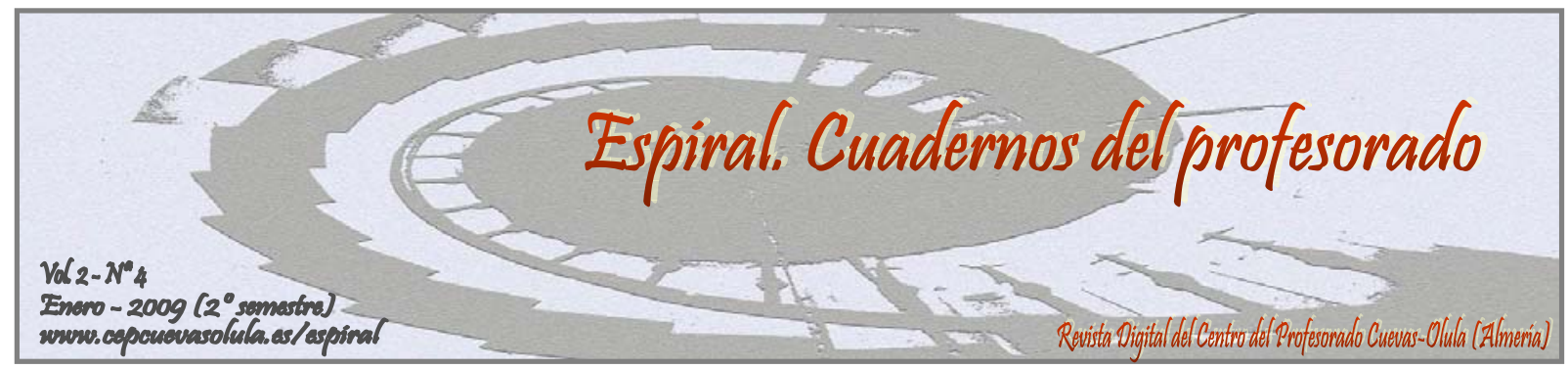

\title{
LA IMPORTANCIA DEL PERÍODO DE ADAPTACIÓN EN EL ALUMNADO DE TRES AÑOS: UNA EXPERIENCIA PRÁCTICA
}

\section{THE IMPORTANCE OF THE PERIOD OF ADAPTATION OF THREE YEAR OLD STUDENTS: A PRACTICAL EXPERIENCE}

\author{
Isabel María Rodríguez Pelegrín
}

\author{
Maestra de Educación Infantil. Centro del Profesorado Cuevas-Olula
}

RESUMEN: En este artículo se describe la experiencia llevada a cabo en un centro durante el período de adaptación por una docente. El objetivo principal es explicar cómo podría planificarse dicho período y ver la importancia que tiene la entrada del niño y la niña de tres años al colegio. Esta debería de hacerse de forma escalonada, respetando sus características, necesidades y sus ritmos de adaptarse al nuevo entorno. Por ello se explica la importancia de que los docentes de la etapa de Educación Infantil, y más concretamente del nivel de tres años, deben de planificar el período de adaptación, adaptarlo a las características del alumnado, con unos objetivos concretos y evaluarlo cuando finalice. Los componentes imprescindibles son: organización, coordinación y una metodología lúdica.

Palabras clave: período de adaptación, entrada escalonada, Educación Infantil.

ABSTRACT: This article describes the experience of a teacher in a educative center during the period of adaptation. The main objective is to explain how to plan this period and point out the importance of the entry of three year old children at school. This should be done in a phased manner, respecting their specific characteristics, needs and rhythms to adapt to new surroundings. Thus explains the importance of teachers in the kindergarten stage, and more specifically in the three year old level, they should plan the adjustment period to adapt to the characteristics of students with specific targets and evaluate when. The essential components are: organization, coordination and playful methodology.

Key words: period of adjustment, staggered entry, nursery school.

Rodríguez Pelegrín, I. M. (2009). La importancia del Período de adaptación en el alumnado de tres años: Una experiencia práctica. Espiral. Cuadernos del Profesorado [en línea], 2(4), 26-33. Disponible en: http://www.cepcuevasolula.es/espiral.

Fecha de recepción: 21/09/2008

Fecha de aceptación: 15/03/2009
Enviar correspondencia a: isapelegrin@gmail.com 


\section{1.- INTRODUCCIÓN.}

En los últimos años se ha producido un cambio sustancial en la sociedad, este cambio abarca muchas parcelas que afectan a la familia, como la incorporación cada vez más numerosa de la mujer al mundo laboral. Ello repercute sin duda alguna, en la educación de los hijos/as, y en consecuencia, en el papel que juega la escuela en este sentido. Por ello, en el año 2002, la Consejería de Educación y Ciencia publicó la Orden de 17 de noviembre de 2003, por la que se convoca a los centros docentes públicos dependientes de la Consejería a solicitar la ampliación del horario de apertura contemplada en el Decreto 137/2002, de 30 de abril, de apoyo a las familias andaluzas, y el Decreto 18/2003, de 4 de febrero, de ampliación de las medidas de apoyo a las familias andaluzas. A partir de ese momento, mucho alumnado pasa a estar más horas en el centro docente que en el hogar, por ello, el objetivo que se persigue es que los centros docentes, más allá de la jornada lectiva tradicional, sean capaces de ofrecer a su alumnado y a las familias una oferta de jornada escolar completa, de forma que encuentren en sus centros las actividades que necesitan para completar su formación y para utilizar de una manera educativa y provechosa su tiempo libre.

Anteriormente a este tipo de modificaciones en los centros, en cuanto a horario, organización...etc. fue todo un reto para el colectivo de infantil, la entrada del alumnado de tres años al centro (LOGSE, 1990), supuso adaptar la programación, la clase, la metodología, etc. y también organizar un período de adaptación.

La adaptación se define como: "Acción y efecto de adaptar o adaptarse", según el Diccionario de la Lengua Española, y si partimos del concepto adaptar, se define en el mismo, de la siguiente manera: "Dicho de una persona: Acomodarse, avenirse a diversas circunstancias, condiciones, etc.".

Esto es lo que le ocurre al niño/a de tres años cuando acude por primera vez al centro escolar, independientemente de que haya estado en la guardería, el centro escolar es una realidad distinta de la que viene y necesita adaptarse a la nueva situación, acomodarse y avenirse a las circunstancias.

Conviene aportar la normativa que se apunta en referencia a este período, para de esta manera ver la importancia que tiene su planificación, desarrollo y evaluación.

Así, el D. 107/1992 de 9 de junio, por el que se establecen las enseñanzas correspondientes a la Educación Infantil en Andalucía, recoge la importancia del período de adaptación (p. 62) destacando:

"Una sensibilidad especial habrá de tenerse en cuenta en los primeros contactos que tiene el alumnado con la escuela. En numerosas ocasiones la entrada en la Escuela de Educación Infantil supondrá para los niños y niñas la primera salida del mundo familiar conocido y seguro, para introducirse en un mundo desconocido y más amplio de relaciones. Debe planificarse cuidadosamente la adaptación personal a un nuevo medio: estableciendo las condiciones materiales, temporales $y$ personales que garanticen una atención individualizada, el respeto al proceso de cada niño o niña, así como la necesaria comunicación con las familias, apoyando afectivamente la resolución de los conflictos que puedan plantearse en este período de adaptación y evitando en cualquier caso situaciones traumáticas”.

Por otro lado en el Real Decreto 1333/1991 de 6 de septiembre por el que se establece el currículo de la Educación Infantil, en el apartado $\mathrm{D}$ del anexo, se hace igualmente referencia a dicho período:"El niño que acude por primera vez a un centro de educación infantil puede encontrarse con que algunos de sus compañeros ha estado ya escolarizados. Cuando ello ocurre, el centro debe planificar la adaptación del niño a su nuevo medio".

Del mismo modo que lo hace Orden de 13 de Mayo de 1999, por la que se regula el calendario y la jornada escolar en los centros docentes, a excepción de los universitarios, en su artículo 4.4:

"A fin de facilitar la escolarización del alumnado de educación infantil que asiste a clase por primera vez y que presente dificultades de adaptación escolar, los Consejos Escolares de los centros podrán establecer, durante el mes de septiembre un horario flexible. Este horario deberá contemplar el tiempo de permanencia de los niños y 
niñas en el centro docente que, de manera gradual y progresiva, será cada día más amplio. En todo caso, a partir del primer día de octubre, el horario de éstos deberá ser el establecido con carácter general para el resto de los alumnos y alumnas de este nivel educativo".

Asimismo, como se expone en el trabajo Lowen (1980):

"cuando un niño pierde la fe en su madre (o su padre) debido a la separación, empieza a perder la fe en sí mismo, porque es la madre la que da seguridad a sus primeros tanteos en la vida, la que le refuerza sus comportamientos, la que da seguridad para el reto que supone la exploración del mundo. Por ello, el tránsito a la escuela es tan importante y la relación con la familia es imprescindible. La entrada al colegio es una primera conquista, de un mundo ajeno a la familia, que requiere una atención especial, mucha ayuda y paciencia. Los cambios importantes de la vida requieren tiempo de elaboración porque debemos, a la vez, ir cambiando nosotros".

Partiendo de estos fundamentos teóricos y basándome en mi propia experiencia, entiendo por período de adaptación, el tiempo que necesita un sujeto que ingresa por primera vez en un colegio, no sólo el niño/niña de tres años, sino todo el alumnado que se escolariza en un nuevo centro, hasta que se integra con el grupo, disfruta de y en el colegio, expresa sus sentimientos, emociones, temores y es capaz de encontrar la magia de la escuela en cualquier momento de la jornada.

De ahí la importancia de programar un período de adaptación. Como docentes debemos de tener un referente normativo y otro práctico para llevar a cabo período de adaptación, y tener un buen equipo y una buena predisposición para llevarlo a cabo como apunta Ibáñez Sandín (1992). Su obra es muy interesante y completa, de hecho yo recurrí al mismo en mis comienzos como maestra, pero con el tiempo había adoptado mi propio "método" para afrontar el "temido" período de adaptación, digo temido porque a todos los colegios a los que me destinaban, las maestras y maestros definitivas en el centro mostraban cierta reticencia a coger al alumnado de tres años, porque eso les suponía tener que adoptar estrategias metodológicas los primeros días para que la clase funcionase con normalidad, muchos docentes desconocían que planificando un buen período de adaptación no se contrarían con ningún problema para el normal desarrollo de su aula.

El período de adaptación debe de planificarse previamente al comienzo del curso. Lo ideal sería empezarlo en mayo o junio, aunque generalmente se hace en septiembre, por causas diversas, como: no saber cuál va a ser tu centro de destino, o qué grupo te asignaran,... no obstante, lo importante es hacerlo, en mayo o septiembre, pero bien planificado y, posteriormente, evaluarlo. Por todo ello, entiendo que el período de adaptación debe de tener objetivos, contenidos, metodología y evaluación.

Antes de pasar a exponer mi experiencia personal, describo los elementos citados anteriormente, siendo éstos flexibles, ya que la realidad de cada centro es distinta y cada niño/niña es único e irrepetible.

\section{2.- OBJETIVOS}

Los objetivos que se deben de plantear han de ser concretos, reales y teniendo siempre como referente las características psicoevolutivas del alumnado de esta edad.

$\checkmark$ Conseguir la adaptación del alumnado al grupo-clase $\mathrm{y}$, posteriormente, al nuevo entorno: el colegio.

$\checkmark$ Aportar al niño/niña estrategias para desarrollar su autonomía en el aula y en el resto del colegio.

$\checkmark$ Desarrollar en cada individuo actitudes positivas hacia el nuevo entorno y la nueva situación a la que se enfrentan.

$\checkmark$ Ayudar a controlar esfínteres.

\section{3.- CONTENIDOS}

La relación de contenidos se refiere a situaciones concretas, significativas para el normal desarrollo del grupo-clase, en general, y de cada sujeto, en particular. Podrían ser:

$\checkmark$ Pautas de comportamiento.

$\checkmark$ Hábitos de higiene, de orden, de colaboración, etc.

$\checkmark$ Cuentos de valores, canciones que expresen emociones, etc. 
$\checkmark$ Dependencias del colegio: servicios, comedor, gimnasio, patio, aula, biblioteca, laboratorio, salón de actos, secretaría, sala de profesores/as...

\section{4.- METODOLOGÍA.}

La metodología que vamos a desarrollar estará basada en los intereses del niño/niña, siendo ésta experimental, significativa, lúdica y motivadora. El eje fundamental va a ser el juego.

El docente utilizará los recursos del juego para, de esta manera, favorecer el desarrollo emocional y social del alumnado, tanto en situaciones normalizadas como en situaciones carenciales.

Durante el período de adaptación se trabajará por grupos, pero, respetando los ritmos individuales de cada alumno/alumna. Se les invitarán a que participen de cuantas actividades se desarrollen en el aula, explicando claramente que no tiene que tener miedo a equivocarse, en esta clase todo se acepta, siempre y cuando se respeten las normas establecidas.

\section{5.- EVALUACIÓN.}

Habrá tres momentos en la evaluación:

- Evaluación inicial: para obtener datos del grupo haremos una reunión con las familias antes de comenzar las clases; en ella se les informará del funcionamiento del centro, de cómo vamos a desarrollar el período de adaptación y, por último, se le entregará un cuestionario para que nos aporten aquellos aspectos significativos del desarrollo de su hijo/hija, etc.

- Evaluación continua: cuando empiece el período de adaptación, observaremos directamente el comportamiento del grupo, en general, y de cada sujeto individualmente, destacando aquellos aspectos más significativos, todo ello lo recogeremos en un anecdotario y en un diario de clase para analizarlo posteriormente.

- Evaluación final: al terminar el período de tiempo que hayamos establecido como de adaptación, volveremos a reunir a los padres/madres para intercambiar informaciones acerca de la actitud de sus hijos/hijas dentro y fuera del colegio. La maestra o maestro hará una evaluación crítica de los mecanismos utilizados, de la metodología, de los objetivos que nos proponíamos al principio así como de los procedimientos y el material utilizado.

\section{6.- MATERIAL.}

Para realizar las actividades dentro del período de adaptación, algunos de los materiales que son necesarios:

- Material fungible: papel, globos, ceras, pintura, música, plastilina, cuentos de valores, disfraces, puzzles, cartulinas,..etc.

- Material no fungible: el propio de un aula de infantil.

- Personal: contar con el docente de apoyo si la hubiese y si tenemos niños/niñas con NEE (necesidades educativas especiales) contaríamos con el monitor/a escolar.

\section{7.- ACTIVIDADES TIPO.}

Las actividades que se describen a continuación, son solo una muestra de las que podrían hacerse en el aula. Estas son las que estarían previstas, pero se pueden incluir las que proponga el alumnado.

- Hacer dibujos sobre la familia, en cartulina, papel, etc.

- Traer fotos de la familia y colgarlas en clase. Cada uno habla de su familia.

- Pintar con las manos, con los pies, con la nariz, etc.

- Cantar y bailar canciones que nos hablen de los sentimientos, etc.

- Maquillaje facial.

- Globoflexia.

- Juegos de socialización.

- Juegos de cooperación.

- Juegos sensoriales.

- Recitar poesías, rimas, adivinanzas, etc.

- Juego por rincones.

- Juegos dirigidos en el patio.

- Lectura de cuentos que ayudan a adaptarse al colegio como los sugeridos por Rovati (2007): “Teo en la escuela”, “Mi primer día de colegio”, "Luisa no quiere comer en el colegio" sobre la primera vez que una niña se queda a comer en el cole, "Lila va al cole" y "En el cole con los Lunnis”, son historias para pequeños de dos o tres años con las se sentirán identificados fácilmente. 
Se pueden hacer cuantas actividades creamos oportunas, tanto planificadas, como las que surjan espontáneamente. Los primeros días deben de tener cabida las proposiciones que nos sugieran los niños/niñas, tienen que ser protagonistas de su propio aprendizaje y de su adaptación. El docente tiene que saber escuchar sus peticiones, dirigir la clase sin autoridad, sin imposiciones, sólo con un cierto orden, para inculcarles hábitos y mostrarles cariño, entusiasmo, respeto, motivación, alegría, bienestar, dulzura, confianza, provocar risas, respetar las rabietas, los enfados y los silencios... Sólo así podremos conseguir que se sientan cómodos en su nuevo espacio, con una nueva figura de referencia, y ello tendrá como repercusión que se adapten a la vida escolar, y habremos conseguido nuestro objetivo, que todos superen el período de adaptación positivamente.

\section{7.- EL PERÍODO DE ADAPTACIÓN EN EL PRIMER CURSO DEL $2^{\text {a }}$ CICLO DE EDUCACIÓN INFANTIL.}

El período de adaptación que se va a describir a continuación, se desarrolló en el CEIP Álvarez de Sotomayor, de Cuevas del Almanzora, Almería, en septiembre del año 2001. Se llevó a cabo con niños y niñas de tres años durante un mes aproximadamente, de clase social media, con un entorno familiar favorable, en la mayoría de los casos, con la implicación del 95\% de las familias, y con el material propio de un aula, pero con la colaboración esencial de la familia y con el deseo y la ilusión de la maestra, de que los niños y niñas se sintiesen cómodos en clase, alegres y al fin y al cabo, FELICES.

Llegué al colegio como maestra provisional, volvía a mi colegio, a reunirme con los docentes que habían formado parte de mi vida escolar. En el claustro en el que se repartían los cursos me asignaron la tutoría de primer nivel de segundo ciclo de Educación Infantil, una de las dos clases de tres años que había en el centro. Era el segundo año que funcionaba una línea de tres años, el curso anterior había sido el primero. Pregunté si se había hecho período de adaptación y una de las compañeras me informó de que se había realizado una entrada escalonada del alumnado.

\section{Planteamiento}

Tras reunirme con mi compañera de nivel, decidimos realizar un período de adaptación, con un objetivo principal, conseguir que la entrada de los niños y niñas de tres años, se hiciera de forma progresiva, planificada, con una programación estructurada, flexible e involucrar a las familias en todo el proceso.

Previamente a realizar la planificación lo consulté con el Equipo Directivo, le expliqué cuales eran las pretensiones que tenía, quería hacer una entrada progresiva de los niños/niñas al aula, pero previamente tenía que reunir a los padres unos días antes, para explicarles en qué consistía el período de adaptación, cual iba a ser su papel en el mismo y como podría repercutir en la entrada al cole de sus hijos/hijas.

Sin impedimento alguno, una vez expuestas mi pretensiones, y contando con la cooperación de la compañera que tenía la otra clase de tres años, decidimos organizar el período de adaptación, a pesar de que estábamos a tan sólo diez días de que comenzase el curso.

Me dispuse, en primer lugar, a llamar a los padres y madres personalmente. Los cité uno a uno, para que asistieran a la reunión una semana antes de comenzar el colegio. Por supuesto que les advertí que tenían que venir sin los niños/niñas, ya que hay veces que subestimamos la capacidad que tienen de escuchar y comprender lo que los adultos hablamos y, en este caso, no era necesario ni conveniente que escuchasen las pautas de comportamiento de las que les iba a hablar a sus familias.

Al principio, cuando comencé a llamar, la tónica general fue la sorpresa de los padres y madres, ya que la mayoría tenían hijos/hijas de edades superiores y anteriormente no se había realizado período de adaptación. No obstante la respuesta fue positiva y un tanto por ciento elevado asistió a la misma, aproximadamente un $70 \%$ del total de padres y madres.

Previamente había preparado los grupos en los que iba a distribuir al alumnado de mi clase para el período de adaptación: había 20 alumnos/alumnas que dividí en tres grupos, dos grupos de siete alumnos/alumnas y uno de seis alumnos/alumnas, con una distribución lo más igualada posible en número, sexo y fechas de nacimiento, ya que no tienen la misma madurez los que nacen a principio de año como los que nacen en la segunda mitad del año, hay una diferencia entre algunos miembros del grupo de casi un año. Concretamente en mi clase contaba con catorce niñas y seis niños.

Los grupos recibieron el nombre de A, B y C, y a cada familia le di la lista del grupo en el 
que estaba su hijo/hija, así como el horario en el que tenía que traerlo al colegio (tabla 1).

Tabla 1. Distribución de grupos y horario de las tres primeras semanas.

\begin{tabular}{cccc}
\hline GRUPO & A & B & C \\
\hline $1^{\text {a }}$ & $9: 00 \mathrm{~h}-$ & $10.30 \mathrm{~h}-$ & $12: 00 \mathrm{~h}-$ \\
SEMANA & $10.30 \mathrm{~h}$. & $12: 00 \mathrm{~h}$ & $13: 30 \mathrm{~h}$. \\
\hline $2^{\mathrm{a}}$ & $12: 00 \mathrm{~h}-$ & $9: 00 \mathrm{~h}-$ & $10.30 \mathrm{~h}-$ \\
SEMANA & $13: 30 \mathrm{~h}$. & $10.30 \mathrm{~h}$. & $12: 00 \mathrm{~h}$ \\
\hline
\end{tabular}

De esta manera, todos los grupos pasarían por todos los horarios, y así los tendrían afianzados, puesto que durante una semana interiorizarían las rutinas que se trabajasen en ese tiempo dentro del aula.

La tercera semana se harían dos grupos, la mitad de la clase en $\mathrm{A}$ y la otra en $\mathrm{B}$, intentando igualar el número de niños y niñas en $\mathrm{A}$ y $\mathrm{B}$, solamente con el siguiente horario (tabla 2).

Tabla 2. Distribución de grupos y horario de la cuarta semana.

\begin{tabular}{|c|c|c|c|c|c|}
\hline GRUPOS & LUNES & MARTES & MIÉRCOLES & JUEVES & VIERNES \\
\hline \multirow{2}{*}{ A } & $9: 00 \mathrm{~h}-$ & 9:00h - & $11: 30 \mathrm{~h}-$ & $11: 30 \mathrm{~h}-$ & 9:00h - \\
\hline & 11:30h & $11: 30 \mathrm{~h}$ & $14: 00 \mathrm{~h}$ & $14: 00 \mathrm{~h}$ & $11: 30 h$ \\
\hline \multirow{2}{*}{ B } & 11:30h- & $11: 30 \mathrm{~h}-$ & $9: 00 \mathrm{~h}-$ & $9: 00 \mathrm{~h}-$ & 11:30h - \\
\hline & $14: 00 \mathrm{~h}$ & $14: 00 \mathrm{~h}$ & 11:30h & $11: 30 \mathrm{~h}$ & $14: 00 \mathrm{~h}$ \\
\hline
\end{tabular}

La intención era que los grupos se fueran mezclando, y pasaran más tiempo dentro del aula. Grupos totalmente flexibles, que variarían en función de la actitud de los propios niños/niñas, del grupo en sí y de la disponibilidad de horario de las familias, ya que algunas familias manifestaban algún problema para traer a sus hijos/hijas a determinadas horas.

A partir de la quinta semana, el grupo vendría al colegio durante toda la jornada escolar, a excepción de que alguien no estuviese adaptado del todo (tabla 3).

Tabla 3. Distribución de grupos y horario de la quinta semana.

HORARIO LUNES MARTES MIÉRCOLES JUEVES VIERNES

\begin{tabular}{cccccc}
\hline De 9:00 a & TODOS & TODOS & TODOS & TODOS & TODOS \\
14 & & & & &
\end{tabular}

\section{Explicación del desarrollo del período de adaptación a las familias.}

Evidentemente, cuando fue planteada esta inserción de los niños/niñas en el colegio, muchas familias se opusieron, a pesar de que fundamenté el por qué y las consecuencias positivas del mismo, sin saber si todo iría como había sido planificado o, por el contrario habría alguna variable que impidiese el buen desarrollo del período.

Les fue explicado y leído literalmente el Real Decreto 1333 de 6 de septiembre de 1991, por el que se establece el currículo de la Educación Infantil, en el apartado D del anexo apunta:

"La llegada del pequeño por primera vez al centro de Educación Infantil debe ser detalladamente planificada. Una cuidada organización del período de adaptación establecerá las condiciones materiales y personales necesarias, para que éste no sea traumático..."

El centro debe poner los medios a su alcance para la adaptación del niño/niña a su nuevo "medio".

También la Orden de 13 de mayo de 1999, que pone de manifiesto: "organizar la "entrada escalonada" de los alumnos/alumnas a la escuela durante los primeros días de clase. Calendario de entrada de los niños/niñas”.

El propósito era fundamentar la importancia del período de adaptación, de lo que podía significar para la toma de contacto de sus hijos/hijas con el colegio, con una persona desconocida para ellos/ellas. Aunque la mayoría de los padres/madres me conocían, muchos se negaron, alegaron que por motivos laborales no podían seguir ese horario. Se les sugirió que lo/la podría traer algún miembro familiar, algún vecino/a, pero fue en vano.

En algunos de estos casos, el alumnado que pasaba toda la jornada escolar durante este período, tuvo más problemas para adaptarse. Veían como entraban y salían el resto de sus compañeros/as y preguntaban por qué no venían a por ellos/ellas, para irse a casa como a por el resto del grupo. Las familias que no llevaron a cabo el período de adaptación vieron día a día la importancia que éste tenía en la entrada del alumnado al nuevo entorno. Cuando llegaban a recoger a este grupo reducido de alumnado, lo primero que hacían los niños/niñas era 
reprocharles lo que habían tardado en ir a recogerlos/las.

Después de fundamentar el por qué de llevar a cabo el período de adaptación, se les comentó que era importante mostrarles a sus hijos e hijas, durante los días previos, confianza en el colegio, transmitirles la alegría que sentían por su próxima entrada en el colegio, no amenazarlos con que cuando fuesen al colegio se iban a "quedar en la gloria", ir con ellos a comprar su primera mochila, que eligiesen la ropa que querían llevar el primer día y, ante, todo no manifestar la ansiedad propia de estas situaciones.

Ese primer día tenían que traer a sus pequeños y pequeñas, y quedarse con ellos/ellas en el aula, para hacer una actividad conjunta. Más tarde se marcharían con una sonrisa en la boca y sin mirar atrás.

La actividad a realizar a lo largo de esa media hora consistía en lo siguiente: la clase estaba preparada con las mesas y las sillas por grupos. Cada uno/a tenía en su mesa su nombre escrito (esto es importante, el niño/niña piensa que todo el mundo sabe cómo se llama y, en este caso, si su maestra/o no sabe el nombre no lo logra entender). La familia, simplemente, habría seguido las instrucciones de la maestra/o y se marcharía cuando le dijese que tenían que ir a comprar una chuchería o regalo, cualquier premio, porque a ella/el se le había olvidado.

\section{Primer día de colegio.}

A las nueve llegó el primer grupo. Algunos/as se mostraron reacios al entrar, pero las madres y padres se metieron totalmente en su papel, llegaron con una sonrisa dibujada en su boca y eso fue decisivo. Me presenté a todos/todas y comencé a contarles la historia del Duende:

"Buenos días a todos, estoy encantada de que os haya tocado venir a este cole, porque en este cole vive mi amigo el Duende, no lo podéis ver, pero él está contentísimo de que los niños/niñas más guapos y guapas hayan venido a su clase...."

El cuento que me había inventado con un poco de imaginación, les daba la bienvenida y hablaba de que al "duende" le gustaba mucho saber cuáles eran sus nombres y el de las personas que los acompañaban; con el cuento les iba dando las instrucciones a las madres y padres, y alguna abuela, sobre lo que tenían que hacer:
- Poner su nombre en un círculo y el de su hijo/hija.

- Colgarlo en el panel de los nombres, que nos había dejado el duende.

- Pegarles la pegatina con su nombre a los niños/as.

- Explicarnos quiénes eran los que aparecían en la foto que había traído de la familia.

- Dejarles su juguete preferido en su sitio.

- Por último, marcharse a la tienda de chucherías a comprarles una golosina.

Resultó de lo más agradable, todos/todas estaban contentos/as, alguno lloró cuando mamá/papá se fue, pero la mayoría no lo hizo, porque seguidamente hicimos una fiesta de bienvenida: hicimos: globoflexia, nos maquillamos la cara... y de pronto vinieron las mamás y los papás a buscarlos. Todo salió más o menos como estaba previsto, a pesar de diferentes factores, como reacciones exageradas de algún niño/niña o despedidas algo dramáticas, el grupo respondió con mucho entusiasmo y aceptaron bien la separación de la persona que les acompañó.

La misma secuencia se fue repitiendo con los tres grupos, y en los días sucesivos se fue alargando el tiempo de cada actividad y acortando la despedida de la persona que los llevaba al colegio.

Y así durante las siguientes tres semanas seguí la estructura explicada. Cuando llegaban se hacía una asamblea, dedicábamos un tiempo a trabajar las rutinas de clase, se hacían juegos dirigidos, descanso, trabajo de hábitos y despedida. Pasadas las cuatro semanas comenzaban con el ritmo normal de una clase de Educación Infantil, en la que apenas interferían rabietas, llantos inconsolables y en la que la armonía y la alegría se respiraban durante toda la jornada escolar

\section{9.- CONCLUSIONES.}

Para concluir este artículo, decir que, en primer lugar aquí solamente se recoge un breve resumen de la planificación y, en segundo lugar, el período de adaptación requiere tiempo de preparación, debe de estar bien planificado, tiene que haber una coordinación con el resto de tutorías de tres años, para seguir todos las mismas pautas de actuación, y por último debemos de evaluarlo cuando finalice, solamente 
de esta manera podremos conseguir que el periodo de adaptación sirva para que los niños y niñas que entran por primera vez al colegio adquieran seguridad, autonomía y desarrollen una actitud positiva en la conquista del nuevo entorno en el que se insertan, porque ello va a suponer la salida del mundo familiar en el que el niño/a se siente seguro para pasar a un entorno desconocido para él/ella, y lo que es más importante a una serie de adultos e iguales con el que va a establecer nuevas relaciones, donde va a variar el rol establecido dentro de la familia y va a ser valorado/a desde otro punto de vista.

La forma en la que tenga lugar esta primera separación del niño con respecto a la familia va a ser fundamental para el proceso de socialización posterior, así como para la actitud posterior hacia las instituciones educativas. En este sentido, destacar que el alumnado que no realizó el período de adaptación como estaba planificado, por diferentes motivos, tuvo más problemas para asimilar esta nueva situación. Las familias que no lo llevaron a cabo, reconocieron que sus hijos/hijas habían manifestado cierto rechazo a la hora de ir al colegio, mientras que el resto lo habían asimilado sin ningún problema.

El conocimiento de las reacciones, comportamientos, sentimientos que surgen en el proceso de separación, va a darnos la comprensión suficiente que posibilitará el tener una actitud serena y confiada que repercutirá en el bienestar emocional también del alumnado.

Para finalizar, espero que sirva esta experiencia para concienciar al profesorado y las familias de que es importante que los cimientos de la educación del alumnado de esta etapa, sean sólidos, son la base de su vida escolar y, por ello, no se pueden dejar al azar, deben de programarse, teniendo en cuenta las características psicoevolutivas, los intereses, las motivaciones $\mathrm{y}$, en definitiva, todo lo que rodea y todo lo que es un niño/a de esta edad.

\section{0. - REFERENCIAS BIBLIOGRÁFICAS.}

Decreto 18/2003, de 4 de febrero, de ampliación de las medidas de apoyo a las familias andaluzas (BOJA n ${ }^{\circ} 26$ de 7 de febrero de 2003). Sevilla.

Decreto 107/1992, de 9 de junio, por el que se establecen las enseñanzas correspondientes a la Educación Infantil en Andalucía (BOJA $\mathrm{n}^{\circ} 20$ de Junio de 1992). Sevilla.
Decreto 137/2002, de 30 de abril, de apoyo a las familias andaluzas. (BOJA $\mathrm{N}^{0} 42$ de 4 de mayo de 2002). Sevilla.

Diccionario de la Real Academia de la Lengua Española [en línea]. Disponible en: http://www.rae.es/ [Consulta: 2008, 4 de abril].

Ley de Ordenación General del Sistema Educativo 1/1990 de 3 de Octubre (BOE, $n^{\circ} 238$ de 4 de Octubre de 1.990). Madrid.

Ibáñez Sandín, C. (1.992):"El proyecto de educación infantil y su práctica en el aula". Madrid: La Muralla.

Orden de 13 de mayo de 1999, por la que se regula el calendario y la jornada escolar en los centros docentes, a excepción de los universitarios (BOJA nº59 de 22 de Mayo de 1999). Sevilla.

Orden de 17 de noviembre de 2003, por la que se convoca a los centros docentes públicos dependientes de la Consejería a solicitar la ampliación del horario de apertura. (BOJA $\mathrm{N}^{\circ}$ 2287 de 26 de noviembre de 2003). Sevilla.

Lowen, A. (1980). “Miedo a la vida”. Buenos Aires. Era Naciente [en línea]. Disponible en: http://perso.wanadoo.es/cgomezmayorga/ publicado/laconquistadelaula.htm.

[Consulta: 2008, 14 de abril].

Real Decreto 1333/1991 de 6 de septiembre, por el que se establece el currículo de la Educación Infantil. (BOE n 216 de 09 de septiembre de 1991). Madrid.

Rovati, D. (2007). "Cuentos que ayudan en la adaptación al cole.”. BEBES y + [En línea]. Disponible en: http://www.bebesymas.com/2007/09/19cuentos-que-ayudan-en-la-adaptacion-alcole. [Consulta: 2008, 4 de marzo].

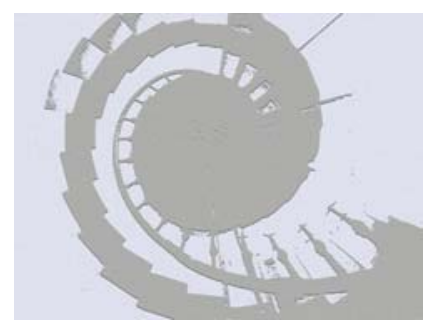

